\title{
Less Phosphorus, More Problems: Hypophosphatemia Induced Polymorphic Ventricular Tachycardia in a Young Male
}

\author{
Robin Jacob ${ }^{1 *}$, Ritesh S. Patel ${ }^{1}$ and Francisco Fuentes ${ }^{2}$ \\ ${ }^{1}$ Resident, Department of Medicine, UT Health Science Center, Houston, Texas, USA \\ ${ }^{2}$ Professor, Cardiovascular Medicine, UT Health Science Center, Houston, Texas, USA
}

*Corresponding author: Robin Jacob, Resident, Department of Medicine, UT Health Science Center, Houston, Texas, USA, Tel: 678-200-6759, E-mail: Robin.jacob@uth.tmc.edu

\author{
Keywords \\ Hypophosphatemia, Phosphorus, Polymorphic ventricular \\ tachycardia, Arrhythmia
}

\section{Introduction}

It is well known that electrolyte derangements such as hypokalemia, hypomagnesaemia, and hypocalcaemia can potentially cause dangerous arrhythmias in hospitalized patients. This can be a common concern for acute care physicians, as the occurrence of arrhythmias predisposes patients to higher morbidity, mortality, and longer length of stay. Phosphorus, however, has been less studied. It is known that hypophosphatemia can disrupt myocardial contractility and reduce cardiac function [1]. Less known, however, is its ability to trigger cardiac arrhythmia. Here we present a rare case of a young male with hypophosphatemia induced polymorphic ventricular tachycardia.

\section{Case Description}

A 23-year-old male with no significant past medical history presented to the emergency department after a mechanical fall. On admission, his vital signs were stable and within normal limits. Physical exam findings included a Glasgow Coma Scale score of 15, bleeding and hearing loss in the left ear, and laceration to the left occipital area. Phosphorus was $2.3 \mathrm{mg} / \mathrm{dl}$ on admission. All other labs, including electrolytes, were normal. Imaging of the head showed small right temporal lobe hemorrhagic contusion. Transthoracic echocardiogram revealed nor- mal left ventricular size and systolic function (ejection fraction 60-65\%) with normal left ventricular diastolic filling and no abnormal findings. Electrocardiogram evidenced normal sinus rhythm.

During his stay, patient's overnight telemetry revealed intermittent, non-sustained ventricular tachycardia (Figure 1). He was asymptomatic at the time, denying any chest pain, shortness of breath, palpitations, or dizziness. Patient denied any personal or family history of heart failure or arrhythmias. Of note, he had not eaten the day prior to the event, and had remained nothing per oral (NPO) on admission. Labs were checked and found to be within normal limits, except for a critically low phosphorus level of $1.4 \mathrm{mg} / \mathrm{dl}$. Arrhythmia continued to persist for several hours until phosphorus was replenished to $3.2 \mathrm{mg} / \mathrm{dl}$, after which the patient no longer had any arrhythmias on telemetry (Figure 2). This patient was on telemetry for his entire hospitalization, and he was discharged several days later with a Holter monitor to evaluate for further episodes of arrhythmia, which was negative.

Cardiac MRI was performed after phosphorus replacement and revealed normal left ventricular function (ejection fraction of $61 \%$ ) with no abnormal late gadolinium enhancement to suggest left ventricular scarring or infiltrative processes. He had no abnormal motion of the anterior free wall of the right ventricle to suggest an arrhythmogenic right ventricular dysplasia/cardiomyopathy.

Given patient's young age, genetic testing was perPolymorphic Ventricular Tachycardia in a Young Male. Int J Clin Cardiol 5:112. doi.org/10.23937/23782951/1410112

Received: April 05, 2018; Accepted: May 10, 2018; Published: May 12, 2018

Copyright: (c) 2018 Jacob R, et al. This is an open-access article distributed under the terms of the Creative Commons Attribution License, which permits unrestricted use, distribution, and reproduction in any medium, provided the original author and source are credited. 


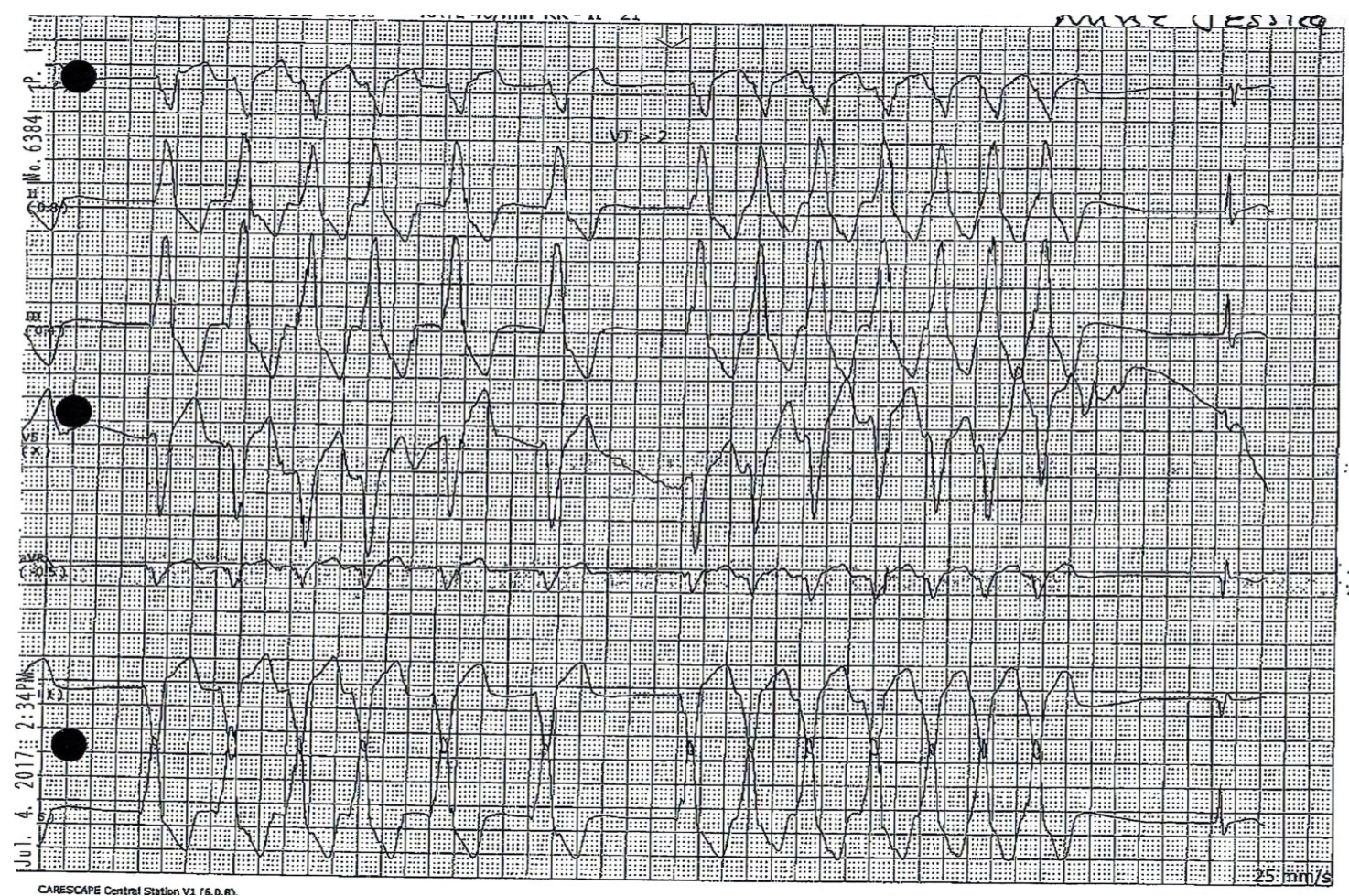

Figure 1: EKG prior to phosphorus replacement.

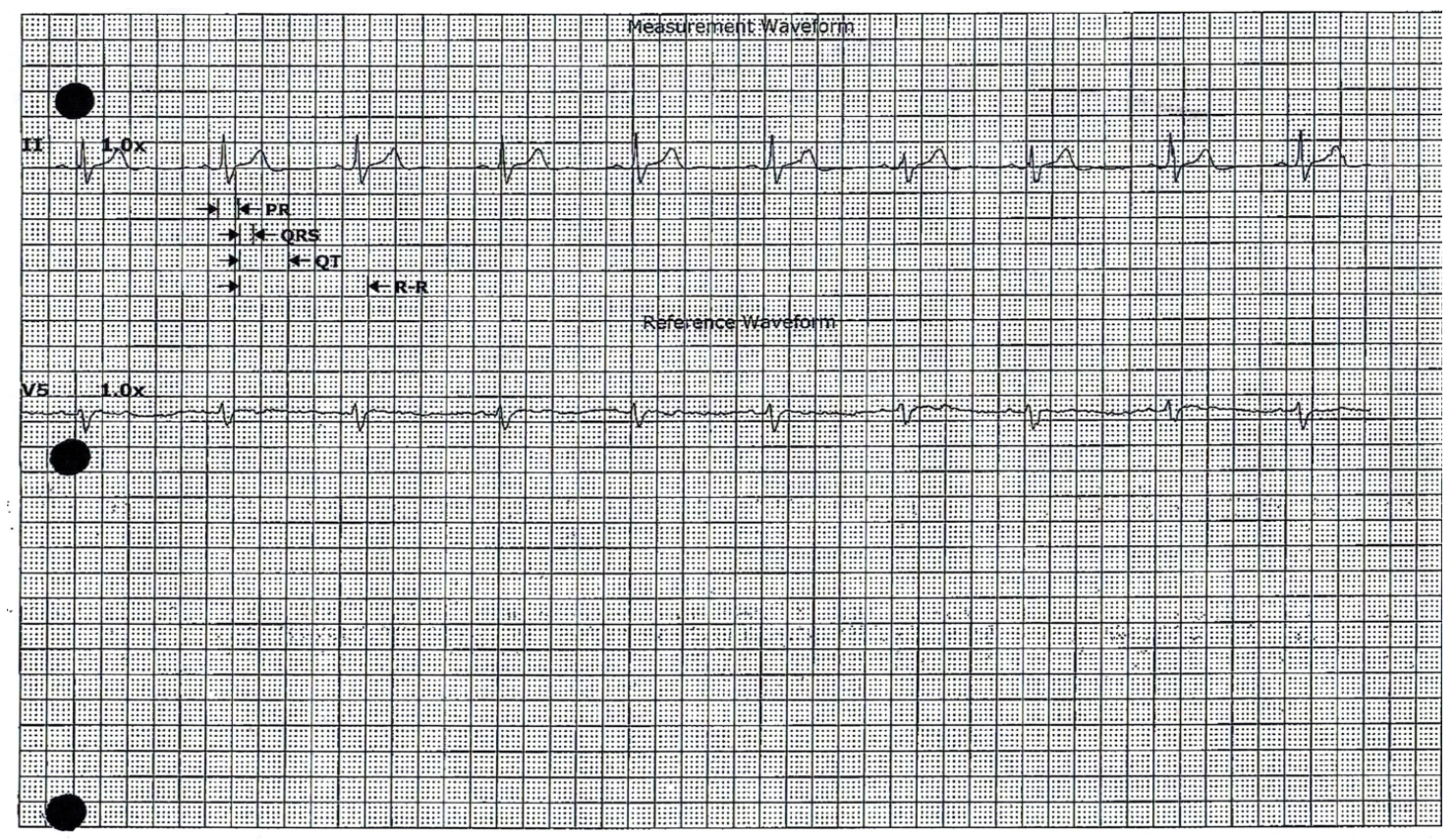

Figure 2: Telemetry strip after phosphorus replacement.

formed with GeneDx Comprehensive Arrhythmia Panel and was found to be negative (Figure 3). This panel tested for $\mathbf{4 6}$ genetic variants associated with arrhythmias such as Long QT Syndrome, Catecholaminergic Polymorphic Ventricular Tachycardia, Arrhythmogenic Right Ventricular Cardiomyopathy, and Brugada Syndrome.

\section{Discussion}

This case illustrates a patient presenting with hypophosphatemia induced non-sustained polymorphic ventricular tachycardia. Delving into the details, phosphorus is an important mineral utilized by almost every cell in the body, with changes in serum values having significant clinical repercussions in patients, especially those who are critically ill [2]. The mineral helps to regulate calcium, another mineral essential for proper cardiac functioning. In addition, phosphorus acts a buffer to help maintain normal acid-base balance, which is important since blood acidity affects heart rate [3]. Phosphorus is also found in 2,3-DPG which helps red blood cells deliver oxygen to the heart [3].

Although fairly rare in the general population, the incidence of hypophosphatemia is high in select groups 
Gene Evaluated: ABCC9, AKAP9, ANK2, CACNA1C, CACN2D1, CACNB2, CALM1, CALM2, CALM3, CASQ2, CAV3, DES, DSC2, DSG2, DSP, GPD 1L, HCN4, JUP, KCND3, KCNE1, KCNE2, KCNE3, KCNE 1L (KCEN5), KCNH2, KCNJ2, KCNJ5, KCNJ8, KCNQ1, LMNA, NKX2-5, PKP2, PLN, RANGRF, RYR2, SCN10A, SCN1B, SCN2B, SCN3B, SCN4B, SCN5A, SNTA1, TGFB3, TMEM43, TRDN, TRPM4, TTN

Result:

\section{No pathogenic variant was detected.}

No pathogenic variants known to be associated with arrhythmia were identified by sequence analysis of the genes on this panel. No deletion or duplication involving any of the genes analyzed was found by concurrent targeted array CGH (ExonArrayDx). The CALM1 gene is not include on Arrhythmia ExonArrayDx.

Interpretation: The Comprehensive Arrhythmia Panel includes sequence and deletion/duplication analysis of genes that cause various arrhythmia syndromes. Many of these genes code for ion channel proteins of the heart muscle that mediate the movement of sodium, potassium and calcium ions in and out of cardiac cells, as well as their associated regulatory factors and interaction partners. The sensitivity of this panel varies depending in part on the individual's clinical phenotype, with the sensitivity being highest in individuals with family history. It is estimated that this panel would detect a pathogenic variant in approximately $40 \%$ of Arrhythmogenic Right Ventricular Cardiomyopathy (ARVC) cases, $15-30 \%$ of Brugada syndrome cases, $45-60 \%$ of Catecholaminergic Polymorphic Ventricular Tachycardia (CPVT) cases, and up to $75 \%$ of LQTS cases (Alders and Christiaans, 2015). Approximately 10\% of individuals with LQTS and no sequence abnormality in one of common LQTS genes have been found to have a large deletion or duplication involving one of these genes (Eddy, et al., 2008).

The frequency of deletions/duplications in genes associated with other arrhythmia disorders such as ARVC, CPVT, and Brugada syndrome is unknown. The percentage of individuals with non-specific arrhythmia that would harbor pathogenic variant(s) detectable by this panel is unknown.

This negative result does not rule out a genetic basis for the arrhythmia phenotype in this individual. It is possible that this individual has a pathogenic variant in a portion of a gene that is not included in this analysis, in a gene that is not included in this arrhythmia panel and/or in an as-yet-unidentified gene associated with arrhythmia.

Recommendation: It is recommended that this individual and any $1^{\text {st }}$ degree relatives receives continued clinical eval and follow up for arrhythmia. Genetic counselling is recommended to discuss the implications of this test report.

Figure 3: GeneDx Panel.

of patients, such as those who are hospitalized (3.1\%) or admitted to intensive care units (33.9\%), and those with sepsis (65 to $80 \%$ ), chronic alcoholism (2.5 to $30.4 \%)$, major trauma (75\%), and chronic obstructive pulmonary disease (21.5\%) [4]. Because of the involvement of phosphorus in every cell of the body, many of the symptoms of hypophosphatemia stem from intracellular phosphate depletion. Symptoms of having low phosphorus include rhabdomyolysis, thrombocytopenia, impaired diaphragmatic contractility, and metabolic encephalopathy resulting from ATP depletion.

Although not as common as seen with hypokalemia, the association between hypophosphatemia and cardiac arrhythmia has recently been made in the literature in septic patients and patients suffering acute myocardial infarction. Dating even further back to the 1970s, studies have looked into the relation of phosphorus to cardiac performance, where patients with severe hypophosphatemia had an increase in left ventricular stroke work, which improved upon return of serum phosphate to normal levels [5]. Mechanism appears to be unknown at this time, but it is postulated that the low phosphate levels may have something to do with the intracellular availability of ATP (3). Because of the importance of phosphorus in cardiac functioning, there have been several studies focused on its association with arrhythmias. In 2014, a study looked at 34 adult septic patients with hypophosphatemia. IV phosphorus replacement was associated with a significantly reduced incidence of arrhythmias ( $38 \%$ vs. $63 \%, p=0.04$ ), demonstrating that phosphorus replacement might be effective in reducing the rate of arrhythmias in septic patients [6]. 
This case occurred in a less critical patient, and as such recognition of the importance of electrolyte replacement in all hospitalized patients is a relevant concern to prevent complications such as arrhythmias.

\section{Conclusion}

Although it is rare, hypophosphatemia should be considered in the differential diagnosis of arrhythmia without an obvious cause. And as always, any other more common causes for arrhythmia, such as hypokalemia or hypomagnesemia, must be corrected prior to attributing the problem to hypophosphatemia.

\section{Sources}

No sources of support.

\section{Conflicts of Interest}

All authors contributed equally to the creation of the manuscript.

\section{References}

1. Ognibene A (1994) Ventricular tachycardia in acute myocardial infarction: The role of hypophosphatemia. South Med J 87: 65-69.

2. Parish, Carol (2013) Low Phosphorus got you down? Nutrition Issues in Gastroenterology.

3. (2018) What does phosphorus do for the heart. Healthy Eating.

4. Gaasbeek A, Meinders E (2005) Hypophosphatemia: An update on its etiology and treatment. Am J Med 118: 1094-1101.

5. Lawrence R, O'Connor, William S, Wheeler, John E Bethune (1977) Effect of Hypophosphatemia on Myocardial Performance in Man. New England Journal Medicine 297: 901903.

6. Schwartz Andrei, Brotfain Evgeni, Koyfman Leonid, Kutz Ruslan, Gruenbaum Shaun, et al. (2014) Association between Hypophosphatemia and Cardiac Arrhythmias in the Early Stage of Sepsis: Could Phosphorus Replacement Treatment Reduce the Incidence of Arrhythmias? Electrolyte Blood Press 12: 19-25. 